\title{
APORTES DE LA MUJER EN LA TRANSFORMACIÓN DE LOS ESTEREOTIPOS SOCIO-CULTURALES DEL DEPORTE COLOMBIANO
}

\section{WOMEN CONTRIBUTIONS TO THE TRANSFORMATION OF THE SOCIOCULTURAL STEREOTYPES OF COLOMBIAN SPORT}

\begin{abstract}
Yanet Lucumí Balanta1
1Licenciada en Educación Física. Magister en Educación. Investigadora Mujer y Deporte. Docente-investigadora Facultad Ciencias del Deporte, Universidad de Ciencias Aplicadas y Ambientales U.D.C.A. Dirección: calle 222 N 55-30, Bogotá D.C. ylucumi@udca.edu.co.
\end{abstract}

Rev. U.D.C.A Act. E Div. Cient. 15 (Supl. Olimpismo): 27 - 35, 2012

RESUMEN

La participación de la mujer en el deporte colombiano se ha visto enmarcada en diferentes estereotipos socio-culturales que, a lo largo de la historia, se han transformando, incentivando a la mujer para vincularse en los diversos rol del deporte, como en la administración, integrando equipos médico-científicos y como deportista, entre otros. La mujer, al participar en los Juegos Olímpicos, demuestra su potencialidad, su empoderamiento e interés deportivo, contradiciendo los imaginarios colectivos negativos que la señalaban como el sexo débil. Entonces, la pregunta de investigación es: ¿Qué aporta la mujer al deporte colombiano y cómo contribuye a la transformación de los estereotipos socio-culturales, con su participación en los Juegos Olímpicos? La mujer deportista olímpica colombiana aporta con su participación en los Juegos Olímpicos, demostrando con resultados deportivos que es capaz de sobrepasar todos los obstáculos que se le presenten, en la consecución de sus metas deportivas (sueños olímpicos) y, con ello, transforma los estereotipos creados, demostrando que sí está interesada en el deporte, ejecutándolo, a nivel deportivo, obteniendo medallas y ocupando posiciones significativas y en otras actividades de tipo administrativas, sociales y culturales, convirtiéndola en ejemplo a seguir.

Palabras clave: Participación, mujer, estereotipos, deporte, Juegos Olímpicos.

\section{SUMMARY}

The participation of women in sport in Colombia has been framed by different socio-cultural stereotypes, which has been transformed throughout history, incentivizing women to connect in this activity from different sport roles, such as administrative tasks, integrating scientific medical teams, and as active sportswoman, among others. Women participating in the Olympics games prove their potential, empowerment and sports memorabilia, thus contradicting the negative collective imagination to it as the weaker sex. Therefore the research question is: how does a woman contributes to Colombian sports and by means of what activity, does she add to the transformation of cultural stereotypes, due to her participation in the Olympics? The Colombian Olympic sport woman contributes due to her participation in the Olympic Games, demonstrating with positive results, that she is capable to overcome all obstacles that are interposed in her goals (Olympic dreams). Therefore, she transforms the stereotypes by pointing out, that if you are interested in sport from different roles, performing them well, achieving medals or occupying significant positions, she will become an example for other women.

Key words: Participation, women, stereotypes, sport, Olympic Games. 


\section{INTRODUCCIÓN}

El presente documento es una recopilación de algunos de los productos de investigación de la línea Mujer y Deporte, liderado por la autora, desde 2006. La información acopiada presenta los aportes de la mujer en la transformación de los estereotipos socio-culturales del deporte colombiano, con su participación en los Juegos Olímpicos. Se tiene como base, los resultados de las investigaciones: factores que han incidido en el posicionamiento de la mujer colombiana de altos logros en el deporte de alto rendimiento, factores asociados a la participación de la mujer bogotana en el juzgamiento deportivo y las historias de vida de aquellas que han participado en los Juegos Olímpicos, desde diferentes roles, para luego confrontarlas con el mito "Las mujeres no están interesadas en el deporte y cuando lo hacen no lo ejecutan bien, como para ser tomadas en serio". Se espera que este artículo tenga un impacto en la comunidad del deporte colombiano, ampliando la visión sobre su participación.

Se considera como problema, a la hora de razonar sobre la participación de la mujer en el deporte que, históricamente, se le negó su participación, como lo menciona Durántez (2001), "bajo pena de muerte", puesto que el instaurador de los Juegos Olímpicos Modernos, el Barón Pierre de Coubertin, así lo estableció, en 1986 y si se atrevían a incumplir dicho mandato serían arrojadas desde el Monte Tipeo. Esta situación cambia 24 años después, cuando la Francesa Alice Millat, en el apoyo de un sinnúmero de colaboradores y colaboradoras, realiza los Primeros Juegos Olímpicos Femeninos, en París, 1922; en Goteborg, 1926 y en Praga, 1930; estos movimientos permitieron la participación femenina en los Juegos Olímpicos de Los Ángeles, en 1932, en pocas disciplinas deportivas, entre ellas, el atletismo. A pesar de esta iniciación, históricamente se ha evidenciado una realidad socio-cultural internacional estereotipada que ha marcado la participación de la mujer en el deporte y, Colombia, no ha estado ajena a dicha situación.

La pregunta de investigación: ¿Qué aporta la mujer al deporte colombiano y cómo contribuye a la transformación de los estereotipos socio-culturales, con su participación en los Juegos Olímpicos?

El objetivo general de la investigación fue, justamente, describir estos aportes y como llevan a pensar, de manera diferente, cuando participan en los Juegos Olímpicos. El estudio, se apoyó en otros fundamentos, como la determinación del deporte de mayor continuidad participativa en Juegos Olímpicos, desde México, 1968 hasta Beijing, 2008 y la descripción de los aportes socio-culturales de las mujeres entrenadoras e integrantes del equipo médico-científico, participantes en Juegos Olímpicos.
Una revisión documental sobre los aportes socio-culturales que hace la mujer en deporte frente a los estereotipos culturales, indica que son escasas las investigaciones ejecutadas al respecto y las existentes, tienen como eje temático su participación en actividad física, a nivel escolar y deportivo, desde otros eventos del ciclo olímpico. Llevar a cabo esta investigación permite vindicar su posición dentro de la sociedad colombiana, contribuyendo a la erradicación de todas las formas de discriminación, como lo menciona las metas del milenio y la declaración de Brighton.

Participación de la mujer colombiana de altos logros en el deporte de alto rendimiento: Se entiende como participación de la mujer en el deporte, como "todas aquellas actividades físicas que buscan a través de la competencia, reglamentada y socialmente reconocida, mejorar un rendimiento y obtener un éxito" (Silva, 2002). Es preciso también indicar que, en términos culturales, su participación ha estado respaldada por diferentes mitos que, de alguna manera, ha afectado su vinculación, como lo señalan Gallo et al. (2002): "a) la actividad deportiva-atlética masculiniza a las mujeres. b) La práctica deportiva es peligrosa para la salud de las mujeres. c) Las mujeres no están interesadas en el deporte y cuando lo hacen no lo ejecutan bien como para ser tomadas en serio"; estos razonamientos surgieron a partir de la participación de la mujer en el deporte Olímpico.

De igual forma, Lucumí et al. (2006), retoman los autores anteriores y mencionan que "la mitología griega asociaba a los dioses con cualidades masculinas, como la fuerza, el vigor y la actividad, mientras que a las diosas, las caracterizaban con rasgos de belleza, de sensualidad y de pasividad. Por tal motivo, las mujeres eran tratadas como porcelanas y desempeñaban un rol de "adorno" pasivo en el deporte, puesto que su fragilidad le impedía desarrollar actividades en las que las capacidades físicas, como la fuerza, la velocidad o la resistencia tenían que ser desarrolladas única y exclusivamente por el hombre -el "sexo fuerte"-, en tanto que la mujer debía conservar su condición de feminidad otorgada por la sociedad".

Marcén (2007) menciona que los niños y los jóvenes deportistas de México tienen la percepción de baja participación de las mujeres y que las causas son: sus padres no las dejan practicar ciertos deportes, en casa no las apoyan, en Educación Física no se les motiva a practicar, les da flojera, les da pena que las vean esforzarse, no se pueden costear los deportes de su interés o prefieren estar en casa. Registró, además, que para los deportistas, el fútbol americano y los deportes de combate son considerados como masculinos y la gimnasia junto con el tenis, como femeninos; los deportes individuales de tiempo y marca presentan una menor estereotipia. También afirma que hombres y mujeres tienen 
capacidades y habilidades diferentes; sin embargo, la mayoría consideran la fuerza "capacidad física que determina el grado de rendimiento deportivo y puede estar influida por el entrenamiento" (Vrijens, 2006) y la agresividad, como propios de hombres y la belleza, la flexibilidad y la sensibilidad, propios de las mujeres.

En Colombia, la participación femenina en el deporte no ha estado alejada de esa realidad mítica cultural internacional. Las mujeres iniciaron su intervención, a nivel competitivo, según el Comité Olímpico Colombiano, en 1938, con la atleta Cecilia Navarrete, en los Juegos Bolivarianos y, en el ámbito Olímpico, en México, 1968, con las nadadoras Carmen Gómez, Olga Lucía de Angulo y Martha Manzano, resaltando que la primera participación colombiana en Juegos Olímpicos fue en Los Ángeles, 1932, con el único deportista, el atleta Jorge "el Perry" Villate.

Esta temática ha sido poco abordada desde las instituciones vinculadas al deporte. Es así como Lucumí $\mathcal{E}$ Jiménez (2009) presentaron como problema que "la práctica del juzgamiento y el arbitraje femenino en Colombia es un fenómeno que culturalmente resulta poco familiar para la hinchada porque tradicionalmente ha sido desarrollado por el género masculino". Los autores identificaron y describieron los factores asociados a la participación de la mujer bogotana en el juzgamiento deportivo: factores personales, del entorno, creencias y capacidad, y determinaron si los factores demográficos tienen relación con los factores asociados. Concluyeron, que en los jueces aún existe la creencia que el desempeño del hombre como juez es diferente al de las mujeres, porque las mujeres involucran sus sentimientos a la hora de tomar decisiones, mientras que los hombres son más parciales; sin embargo, existe una postura diferente y positiva con relación a su desempeño laboral. Los autores también indicaron que las relaciones personales entre jueces y deportistas son cordiales, respetuosas, son vistas como autoridad, a pesar que algunas de ellas se sientan inseguras a la hora de tomar decisiones, en especial, si son jóvenes; además perciben algún tipo de influencia dependiendo si se es hombre o mujer, a la hora de ejercer su labor, fundamentalmente, en algunas disciplinas deportivas en las que debe expresar, de manera verbal, cualquier decisión.

Por otra parte, el Instituto Colombiano del Deporte (COLDEPORTES), en convenio con la Universidad Santo Tomás adelantaron una investigación titulada "Posicionamiento de la mujer colombiana de altos logros en el deporte de alto rendimiento", cuyo objetivo era identificar los factores que habían incidido en el posicionamiento de la mujer colombiana de altos logros en el deporte de alto rendimiento, desde 1992 hasta 2006, los cuales, según Lucumí et al. (2006) fueron: "apoyo incondicional de la familia, fogueo internacional, patrocinio del Estado a través de programas deportivos, patrocinio de la empresa privada, factor socio-cultural, características individuales, condición fisiológica, entrenamiento con los hombres y edad deportiva", lo que pone en tela de juicio dichos mitos o imaginarios colectivos inmersos en la sociedad colombiana, en especial, el que dice "Las mujeres no están interesadas en el deporte y cuando lo hacen no lo ejecutan bien como para ser tomadas en serio". Los resultados de dicha investigación en el factor socio-cultural concluyen que las mujeres colombianas han venido aumentando su participación y resultados deportivos, en términos de medallas, en los eventos del ciclo Olímpico y, principalmente, en Juegos Olímpicos, contribuyendo en la transformación de los estereotipos socio-culturales, a nivel deportivo, pues en palabras del Consejo Superior de Deportes (2001), "la participación de la mujer en el deporte ha ido cambiando a medida que ha ido cambiando su rol social" .

Al hacer referencia a los estereotipos es preciso aclarar la existencia de prejuicios, que Worchel et al. (2002) definen como "actitudes y opiniones, por lo general negativas respecto de los miembros de un grupo. Son la evaluación de alguien basada únicamente en raza, sexo, religión o a que forma parte de otro grupo". Por otro lado, continúan mencionando los autores que desde los factores motivacionales de los prejuicios, éstos "son el resultado de tensiones, emociones, miedos y necesidades elementales del sujeto. En particular, los prejuicios y la discriminación sirven para reducir estados emocionales negativos o satisfacer necesidades básicas". Los prejuicios junto con los estereotipos "dan lugar a la discriminación, la conducta dirigida a alguien únicamente por pertenecer a determinado grupo", por lo tanto, la discriminación hace que exista favoritismo o discriminación positiva. Mencionan los mismos autores, que la discriminación, se puede observar a través de los actos manifiestos de las personas hacia los miembros de los grupos y que cuando la discriminación se basa en los prejuicios, desde el sexo, se llama sexismo y se califica de sexista a quien la evidencia. Lucumí \& Ortiz (2010) definen los prejuicios como "aquellas actitudes cargadas de emociones negativas hacia un grupo determinado de personas, en donde junto con los estereotipos dan lugar a la discriminación positiva, es decir, al favoritismo, en donde se aprueba todo a las personas que pertenecen al grupo de quien discrimina y negativa al ser actos que dañan a los demás"

Para Worchel et al. (2002), las teorizaciones han identificado un grupo de factores motivacionales acerca de los prejuicios y señalan que los prejuicios, desde este referente "son el resultado de tensiones, emociones, miedos y necesidades elementales del sujeto. En particular, los prejuicios y la discriminación sirven para reducir estados negativos o satisfacer necesidades básicas". De igual forma, es importante con- 
ceptualizar los estereotipos y según el mismo autor, el primer proceso que lleva a los estereotipos es el acto perceptual puro de la categorización. Categorizar significa, entonces, colocar en un grupo a un objeto, a una persona, a un animal, identificar lo que es, es decir, identificar a qué categoría pertenece. La categorización es un proceso cognoscitivo básico y automático que cumple una enorme función de adaptación en nuestros esfuerzos por enfrentar un mundo complicado. Al categorizar los objetos, estamos en posición de hacer suposiciones acerca de cómo serán los estímulos.

Así como se categorizan los objetos, también se categorizan las personas en grupos: por género, por raza, por profesión, por ocupación, por deporte, por edad, por región, entre otros y se hacen también suposiciones frente a los comportamientos o características de cada uno de ellos. Afirman Worchel et al. (2002) que: "La categorización de las personas en grupos tiene algunas consecuencias cognoscitivas inmediatas e importantes. Una es que magnificamos o acentuamos las diferencias entre los miembros de grupos diversos. Por eso nos parece que las personas de dos grupos son muy diferentes. Segunda, minimizamos las diferencias entre los individuos que forman parte del mismo grupo; sin embargo, la medida en que lo hagamos depende del tipo de grupo. Nuestras percepciones de los grupos a los que pertenecemos —nuestros grupos — son menos homogéneas (más diversas) que nuestras impresiones de los grupos que no son los nuestros —los ajenos_".

Estos mismos autores mencionan que los estereotipos y las convicciones sobre las características de los miembros de los grupos propios y ajenos, se originan en las familias, gracias al ejemplo, la enseñanza de los padres y de las instituciones, son considerados como las fuentes socio-culturales. Así pues, es en la familia donde se fortalece lo masculino y lo femenino. Cajiao Restrepo (1997) manifiesta que "a lo largo de la historia las diferentes culturas humanas han dado un nivel de significación particular al sexo, asignando funciones y comportamientos altamente diferenciados para hombres y mujeres", con el objetivo de asegurar la supervivencia del grupo, además esta actividad es transmitida de generación a generación.

En cuanto a la participación de la mujer en otros roles distintos al deporte, Sarmiento \& Vargas (2002) señalan las percepciones de las mujeres sobre los cambios en el trabajo, manifestando que "la crisis ha llevado a las mujeres a buscar nuevos espacios de actuación, darse cuenta que sí pueden realizar acciones en el ámbito productivo y valorar su capacidad de aporte económico, por lo que han elevado su autoestima y autonomía”. Del mismo modo, señalan que "las mujeres jóvenes de estratos socio-económicos medios y altos perciben una mejor situación laboral respecto a generaciones anteriores", pero, finalmente, "se requiere de una mayor conciencia social" y, en especial, de los hombres, para valorar más y de manera equitativa todas la actividades que tienen que desarrollar a nivel laboral y familiar.

\section{MATERIALES Y MÉTODOS}

Es un ensayo descriptivo que, bajo la percepción de Hernández et al. (2007) "busca especificar las características" de los aportes que la mujer deportista colombiana ha hecho frente a los estereotipos socio-culturales, desde su participación en Juegos Olímpicos. El enfoque es cualitativo, porque presupone una realidad dinámica dentro del deporte colombiano. Se utilizó la entrevista, como Instrumento de investigación. La población corresponde a las deportistas, administrativas deportivas e integrantes del equipo médico-científico deportivo, que han participado en Juegos Olímpicos, desde 1968 hasta 2008. La muestra correspondió a 15 deportistas, cuatro Fisioterapeutas, asistentes a Juegos Olímpicos y una entrenadora deportiva de alto rendimiento olímpico. Los criterios de inclusión, se centraron en haber participado o sido entrenadora de deportistas olímpicas en Juegos Olímpicos, desde 1968 hasta 2008.

En los criterios de exclusión estaba el no haber participado o sido entrenadora de deportistas en Juegos Olímpicos, desde 1968 hasta 2008. A las mujeres integrantes de la muestra se les informó sobre el proyecto y, de manera voluntaria, decidieron hacer parte de éste, por ello, se continuó con la fase de aplicación de la entrevista.

Por otro lado, se revisaron los archivos del Comité Olímpico Colombiano frente a la participación de la mujer deportista en Juegos Olímpicos, para hacer el diagnóstico participativo.

\section{RESULTADOS Y DISCUSIÓN}

Las 15 deportistas entrevistadas participantes en Juegos Olímpicos tenían como objetivo ser selección Colombia en este evento; para ello tuvieron que trabajar muy fuerte soportando, según Suay (2003) dobles "cargas de entrenamiento" diarias, divididas en dos jornadas; algunas, se vieron obligadas a vivir en ciudades lejos de sus sitios de origen, separándose de sus familias, para poder contar con un mejor entorno de entrenamiento y así mejorar sus resultados deportivos. A pesar de los obstáculos deportivos, emocionales y motivacionales (intrínsecos y extrínsecos) que se les presentaron, siempre contaron con personas cercanas a ellas, quienes les ayudaron a cumplir con sus metas deportivas.

El hecho de participar en los Juegos Olímpicos reflejó en ellas un sentimiento de felicidad, por cumplir su sueño de- 
portivo, además, que se convencieron que el talento deportivo, la disciplina y el esfuerzo, rodeados de un entorno de entrenamiento y de unas condiciones básicas de vida suplidas, son fundamentales para participar en los Juegos Olímpicos y que no es un imposible para los deportistas colombianos. Esto confirma que el deporte es una "fuente de superación" como lo señala el Comité Olímpico Español (s.f.).

En los datos revisados en el Comité Olímpico Colombiano (2000) frente a la participación de la mujer deportista en Juegos Olímpicos, se halló que ha aumentado de manera progresiva, de 3 a 22 participantes (Cuadro 1). De manera similar, frente a los logros deportivos de las mujeres en Juegos Olímpicos, pasaron de medalla de bronce, en 1992, a medalla de oro, en el 2000, en un rango de ocho y tres medallas más de bronce, en los dos siguientes Juegos Olímpicos: Atenas 2004 y Beijing 2008, en deportes considerados para hombres (Levantamiento de Pesas, Ciclismo y Judo) (Cuadro 2).
Si se retoma el imaginario colectivo, "las mujeres no están interesadas en el deporte y cuando lo hacen no lo ejecutan bien como para ser tomadas en serio", se cae de peso, puesto que las mujeres colombianas sí están interesadas en el deporte; ello se demuestra en los Juegos Olímpicos de México 1968, donde participaron tres deportistas en la disciplina de natación. En el tiempo recorrido entre éstos juegos y los realizados en Atlanta 1996, las disciplinas deportivas con asistencia fueron atletismo, natación, ciclismo y tiro. A partir de los Juegos Olímpicos de Sydney 2000, aumentan los deportes de participación femenina por Colombia, pasando de una disciplina deportiva, en la década de los 90, a once disciplinas deportivas y 22 deportistas en el nuevo siglo, lo cual, se podría interpretar: primero, como un cambio cultural en las nuevas generaciones frente a los estereotipos socio-culturales y, segundo, como fruto de un modelo a seguir de aquellas deportistas que abrieron la brecha dentro del deporte colombiano, como María Isabel Urrutia, Eucaris Caicedo, Amparo Caicedo, Luz Mery Tristán, entre otras.

Cuadro 1. Participación de la mujer colombiana en Juegos Olímpicos, desde México 1968 hasta Beijing 2008.

\begin{tabular}{|c|c|c|c|}
\hline $\begin{array}{c}\mathrm{N}^{\circ} \\
\text { PARTICIPACIÓN }\end{array}$ & OLIMPIADA & DEPORTE & PARTICIPANTES \\
\hline 1 & México 1968 & Natación & Olga Lucía de Angulo, Carmen Gómez, Martha Manzano. \\
\hline 2 & Munich 1972 & Atletismo & Juana Mosquera, Elsy Rivas. \\
\hline 3 & Los Ángeles 1984 & Tiro & Alejandra Hoyos, Gloria López, Elvira Carrasquilla. \\
\hline 4 & Barcelona 1992 & $\begin{array}{l}\text { Atletismo } \\
\text { Tiro }\end{array}$ & $\begin{array}{l}\text { Amparo Caicedo, Norfalia Carabalí. Olga Escalante, Isabel } \\
\text { Urrutia, Ximena Retrepo. } \\
\text { María V. Echavarría. }\end{array}$ \\
\hline 5 & Atlanta 1996 & $\begin{array}{l}\text { Atletismo } \\
\text { Ciclismo contra } \\
\text { reloj } \\
\text { Natación }\end{array}$ & $\begin{array}{l}\text { Mirtha Broock Forbes, Sandra Borrero, Patricia Rodríguez } \\
\text { Orejuela, Felipa Alicia Palacios Hinestroza, Ximena Restrepo } \\
\text { Gaviria, Zuleima Aramendiz Mejía. } \\
\text { Maritza Corredor. } \\
\text { Isabel Cristina Ceballos Rojas. }\end{array}$ \\
\hline 6 & Sydney 2000 & $\begin{array}{l}\text { Atletismo } \\
\text { Ciclismo } \\
\text { Ciclo-montañis- } \\
\text { mo } \\
\text { Clavados } \\
\text { Esgrima } \\
\text { Natación } \\
\text { Levantamiento } \\
\text { de pesas }\end{array}$ & $\begin{array}{l}\text { Digna Luz Murillo Rivas, Felipa Alicia Palacios Hinestroza, } \\
\text { Mirtha Brock Forbes, Ximena Restrepo Gaviria, Yanet } \\
\text { Lucumí Balanta, Norma González Camilde, Sabina Moya } \\
\text { Rivas, Iglandini González Pardo. } \\
\text { Maria Luisa Calle Williams } \\
\text { Marina Delgadillo Ruíz } \\
\text { Diana Isabel Pineda Zuleta } \\
\text { Ángela María Espinosa Toro } \\
\text { Isabel Cristina Ceballos Rojas } \\
\text { Carmenza Delgado Castillo, } \\
\text { María Isabel Urrutia Ocoró. }\end{array}$ \\
\hline
\end{tabular}


Continuación cuadro 1.

\begin{tabular}{|c|c|c|c|}
\hline $\begin{array}{c}\mathrm{N}^{\circ} \\
\text { PARTICIPACIÓN }\end{array}$ & OLIMPIADA & DEPORTE & PARTICIPANTES \\
\hline 7 & Atenas 2004 & $\begin{array}{l}\text { Atletismo } \\
\text { Ciclismo } \\
\text { Esgrima } \\
\text { Judo } \\
\text { Levantamiento } \\
\text { de pesas } \\
\text { Natación } \\
\text { carreras } \\
\text { Taekwondo } \\
\text { Tenis campo } \\
\text { Tiro } \\
\text { Triatlón }\end{array}$ & $\begin{array}{l}\text { Melissa Murillo Rivas, Felipa Alicia Palacios Hinestroza, } \\
\text { Digna Luz Murillo Rivas, Norma González Camilde, Zuleima } \\
\text { Aramendiz Mejía, Katherine Ibargüen, Sandra Patricia } \\
\text { Zapata Portela, Darlenis Obregón Mulato. } \\
\text { María Luisa Calle Williams. } \\
\text { Ángela María Espinosa Toro. } \\
\text { Lisset Johana Orozco. } \\
\text { Mabel Mosquera Mena Ubaldina Valoyes Cuesta, Ángela } \\
\text { Medina. } \\
\text { Paola Duguet Durán. } \\
\text { Gladys Alicia Mora Romero. } \\
\text { Fabiola Zuluaga Amado. } \\
\text { Amanda Mondol Cuellar. } \\
\text { Fiorella D’Croz Brusatin. }\end{array}$ \\
\hline 8 & Beijing 2008 & $\begin{array}{l}\text { Arquería } \\
\text { Atletismo } \\
\text { Ciclismo de pista } \\
\text { Gimnasia } \\
\text { Levantamiento } \\
\text { de pesas } \\
\text { Lucha } \\
\text { Natación } \\
\text { carreras } \\
\text { Clavados } \\
\text { Taekwondo } \\
\text { Tenis de mesa }\end{array}$ & $\begin{array}{l}\text { Ana María Rendón Martínez, Natalia Sánchez Echeverri. } \\
\text { Bertha Oliva Sánchez, Eli Johana Moreno, Darlenis } \\
\text { Obregón Mulato, Rosibel García Mena, Yomara Hinestroza } \\
\text { Murillo, Sandra Patricia Zapata Portela, Zuleima Aramendiz } \\
\text { Mejía. } \\
\text { María Luisa Calle Williams. } \\
\text { Natalia Sánchez Cárdenas. } \\
\text { Leidy Yesenia Solis Arboleda, Mercedes Isabel Pérez Tigre- } \\
\text { ros, Tulia Ángela Medina Alcalde, Ubaldina Valoyes Cuesta. } \\
\text { Jacqueline Rentería Castillo. } \\
\text { Erika Layn Steward, Carolina Colorado Henao. } \\
\text { Diana Isabel Pineda Zuleta } \\
\text { Gladys Alicia Mora Romero, Doris Esmid Patiño Marín. } \\
\text { Paula Andrea Medina. }\end{array}$ \\
\hline
\end{tabular}

Cuadro 2. Medallería olímpica femenina.

\begin{tabular}{|l|l|l|l|}
\hline \multicolumn{1}{|c|}{ MEDALLISTAS OLÍMPICAS } & \multicolumn{1}{|c|}{ OLIMPIADA } & \multicolumn{1}{c|}{ DEPORTE } & \multicolumn{1}{c|}{ MEDALLA } \\
\hline Ximena Restrepo Gaviria & Barcelona & Atletismo & Bronce \\
\hline María Isabel Urrutia Ocoró & Sydney & Levantamiento de pesas & Oro \\
\hline Mabel Mosquera Mena & Atenas & Levantamiento de pesas & Bronce \\
\hline María Luisa Calle Williams & Atenas & Ciclismo & Bronce \\
\hline Jacqueline Rentería Castillo & Beijing & Lucha & Bronce \\
\hline
\end{tabular}

A continuación, se presentan dos respuestas de la entrevista realizada a las deportistas olímpicas: "entendí que si había escogido el atletismo como profesión era porque soy una persona privilegiada, con un regalo hermoso, el talento deportivo y al saber que sí podía hacerlo, me dediqué a él por completo. Quise practicar Atletismo, porque quería ser y 
seguir el ejemplo de atletas nortecaucanas, como Norfalia Carabalí, Yanet Lucumí y Norma González". Otra respuesta: ..."ahí inicia mi historia, en la clase de atletismo realizaron unas competencias y quedé de segunda y alguien me dijo que la niña que ganó entrenaba ahí todo los días, eso me motivó a iniciarme también como deportista y empecé a entrenar con el profesor Julio César Abadía, en octubre de 1989".

El aumento participativo femenino deportivo en Colombia, a partir de Sydney, se refleja a nivel internacional, pues fueron definidos, según el Consejo Superior de Deportes (2001), como "los Juegos de la mujeres", por pasar de una participación de cero, en 1896, entre un total de 280 y ascender a 4.028, entre un total de competidores de 10.626. Del mismo modo, la Comisión de Mujer y Deporte del Comité Olímpico Español manifiesta el aumento participativo de sus deportistas, en donde pasan de 22, de un total de 997 competidores, en la primera participación femenina, celebrada en Paris 1900, a 4.069, de un total de 10.651 competidores seleccionados para los Juegos Olímpicos de Sydney 2000. Al extender la participación hasta Beijing 2008, se observa que participaron 4.746 españolas, de un total de 11.196 seleccionados. Este aumento, se ve reflejado también a nivel laboral en el trabajo de las mujeres en Colombia (Sarmiento $\varepsilon$ Vargas, 2002) aunque en estos roles se ve más marcado el machismo por una "cultura patriarcal".

De lo expuesto, se concluye que a lo largo de los 40 años de participación femenina colombiana en los Juegos Olímpicos, se podría decir que se han transformado los estereotipos socio-culturales y, en especial, porque se evidencia el aumento progresivo de la mujer dentro del deporte y su interés en diferentes disciplinas deportivas, incluso, en aquellas que eran consideradas sólo para hombres, como tiro, esgrima, levantamiento de pesas y deportes de combate.

Es importante recordar que el levantamiento de pesas inició su primera participación como disciplina deportiva en los Juegos Olímpicos de Sydney 2000, en donde se obtuvo la primera medalla de oro colombiana, la que fue merecida por la deportista María Isabel Urrutia Ocoró; este deporte tuvo continuidad en posiciones de medallería en los Juegos Olímpicos de Atenas 2004, con Mábel Mosquera, quien fue premiada en el pódium con la medalla de Bronce, al igual que María Luisa Calle Williams, en ciclismo, una disciplina deportiva en la que ha tenido que luchar contra la inequidad y no visibilidad, según Lucumí et al. (2006).

De igual forma, la Lucha Olímpica, otra disciplina deportiva considerada para hombres, adiciona el aporte femenino para la transformación de estereotipos en el deporte colombiano, con la deportista Jacqueline Rentería, quien es medallista de
Bronce en los Juegos Olímpicos de Beijing 2008, demostrando una vez más con sus resultados deportivos que el deporte no tiene género y que las capacidades condicionales de fuerza y de resistencia, asignadas de manera exclusiva para los hombres, desde la mitología Griega, se encuentran presentes en ambos géneros y que de igual forma pueden llegar a maximizarse los resultados deportivos.

Algunas de las respuestas de las deportistas entrevistadas que evidencian la presencia de estereotipos y aportes de éstas son: a) “....además una de las cosas que más me impulsó a hacerlo es que en Cali escuché el siguiente comentario "tan ilusa pensar que llegaría a asistir a Juegos Olímpicos"; b) ¿Qué obstáculos se presentaron? “...todos, desde el desprestigio por ser una mujer de 35 años de edad... isi la edad no es la que compite!; recuerdo que me lesioné el año anterior a los Juegos Olímpicos, en ese momento me di cuenta que en el deporte sí existe la discriminación por edad y rendimiento, lo más duro es que no te dan la oportunidad para demostrar que eres bueno y que la edad es lo de menos". c) "...Ser campeona Olímpica me ha permitido ser parte del Congreso de la República y desarrollar competencias administrativas sumadas a la experiencia deportiva y desde allí aportar en la reforma a la ley 181, en lo relacionado con los Incentivos Deportivos, pues la realidad de los deportistas colombianos es que no todos van a tener la dicha de ganar una medalla Olímpica, como los que hemos llegado a tan grande logro deportivo; mi legado es que ahora los deportistas campeones Centroamericanos, Panamericanos, Mundiales y Olímpicos, inmediatamente sean campeones pueden recibir su incentivo económico y no tendrán que esperar a cumplir los 50 años para disfrutar de ello".

Se concluye, también, que el atletismo ha sido el deporte de mayor continuidad en Juegos Olímpicos, pues de las ocho olimpiadas de participación femenina (México 1968, Munich 1972, Los Ángeles 1984, Seúl 1988, Barcelona 1992, Atlatanta 1996, Sydney 2000, Atenas 2004 y Beijing 2008), ésta disciplina ha hecho parte de la selección Colombia en siete ocasiones, de lo que se podría decir que es una disciplina de alto interés para las mujeres colombianas por la facilidad de acceso, pues los recursos que se requieren para la práctica de la misma son poco costosos. Otro factor es el reconocimiento deportivo que tienen las atletas colombianas, pues sus logros deportivos motivan a otras a seguir esta actividad. Por otro lado, la mujer colombiana hoy se ha vinculado al deporte en diferentes roles, como administradora deportiva, juez, entrenadora con deportistas Olímpicos, haciendo parte del equipo médico-científico, como fisioterapeutas, sicólogas y nutricionistas, desmintiendo así los estereotipos alusivos frente a su participación en el deporte y para ellas, en especial, para estas profesionales, es un triunfo significativo, porque han venido posicionándose altamente, demostrando 
con su trabajo que son importantes para el bienestar físico y emocional de los deportistas, ya que se encargan de la recuperación deportiva, minimizando las dolencias musculares de los participantes y tratando las lesiones presentes en ellos (recuperación), en palabras de Silva (2002), "el proceso por medio del cual se busca restablecer la capacidad físico - atlética y psicológica, luego de un esfuerzo".

El papel que juegan las Sicólogas permite que los deportistas conserven su confianza y motivación; lamentablemente, tienen que demostrar ante entrenadores, médicos y directivos del deporte, que su labor no es fácil y mucho más por ser mujer. En el caso de las Entrenadoras Deportivas Olímpicas, "persona experta que motiva, entrena y dirige en competencias un deportista o equipo deportivo. A su formación en Educación Física debe sumarse los conocimientos en un deporte específico y conocimientos en teoría del entrenamiento deportivo" (Silva, 2002), ha sufrido tratos de discriminación, pues los imaginarios sociales enmarcaban estas actividades como exclusivas para los hombres, pero, en Colombia, la mujer entrenadora, también ha llevado deportistas a Juegos Olímpicos, aunque su trabajo ha sido opacado por la cultura machista.

Finalmente, frente a la pregunta: ¿Qué aporta la mujer al deporte colombiano y cómo contribuye a la transformación de los estereotipos socio-culturales, con su participación en los Juegos Olímpicos?, se concluye que sus logros deportivos no son solo en términos de medallería sino también a nivel participativo como deportista, como entrenadora y como integrante del equipo médico-científico; son un ejemplo a seguir para las generaciones y, que tanto hombres como mujeres, las admiran, las respetan y valoran su trabajo, porque han demostrado que no se trata de género sino de capacidad volitiva (voluntad) y capacidad de cambio en el pensamiento de sí mismas y en los otros, generando transformaciones generacionales en la cultura deportiva colombiana.

Se han venido transformando los estereotipos deportivos en Colombia, porque son más la mujeres que hoy participan en el deporte olímpico, en 1968 fueron tres deportistas, en Beijing 2008 fueron 22 y sei s entre administrativas, delegadas deportivas e integrantes del equipo médico-científico. Se espera que para la participación femenina olímpica por Colombia, número 44, a celebrarse en Londres 2012, se superen estas cifras de asistencia y que exista igualdad de oportunidades para ambos géneros.

Conflicto de intereses: La autora declara que no existe ningún conflicto de intereses que ponga en riesgo la validez de la información presentada.

\section{BIBLIOGRAFÍA}

1. CAJIAO RESTREPO, F.A. 1997. La piel del alma. Cuerpo, educación y cultura, Magisterio. Bogotá. p.363.

2. COMITÉ OLÍMPICO COLOMBIANO. 2000. 100 años de la mujer en los Juegos Olímpicos. Sydney 2000. No. 3. p.33.

3. COMITÉ OLÍMPICO ESPAÑOL. s.f. Manual técnico y colegios pdf. Campaña de difusión de los Juegos Olímpicos. Oficina de atención al deportista. (España). 3p. disponible desde internet en http://www.oad.es/oad/ gesOAD.nsf/0/C9F777F5C584F7CEC12573FA0040 09CB/\$File/manual\%20tecnico\%20y\%20colegios.pdf (con acceso 15/01/ 2012).

4. COMITÉ OLÍMPICO ESPAÑOL. s.f. Participación femenina Española en Juegos Olímpicos. Disponible desde internet en http://www.mujerydeporte.org/org/jjoo/ jjoo_verano.asp (con acceso 09/02/2012).

5. DURÁNTEZ, C. 2001. Historia y filosofía del olimpismo, Comité Olímpico Español. Academia Olímpica Española.

6. GALLO, L.; CASTRO, J.; OCHOA, V.; MONSALVE, O. 2002. Mujer y Deporte en Medellín y su Área Metropolitana. Rev. Des-Encuentros 1(5):5-13.

7. HERNÁNDEZ SAMPIERI, R.; FERNÁNDEZ COLLADO, C.; BAPTISTA LUCIO, P. 2007. Fundamentos de metodología de la investigación. McGraw-Hill (España). 60p.

8. LUCUMÍ, Y.; CASALLAS, P.; PARRA, F. 2006. Posicionamiento de la mujer colombiana de altos logros en el deporte de alto rendimiento desde 1992 hasta el año 2006. U. Santo Tomás. Coldeportes. p.8-9.

9. LUCUMÍ, Y.; JIMÉNEZ H. 2009. Factores asociados a la participación de la mujer bogotana en el Juzgamiento deportivo. U. Santo Tomás. p.37, 53.

10. LUCUMÍ, Y.; ORTÍZ, R. 2010. Comprensión de los componentes educativos en equidad de género en el ciclo básico del campo físico deportivo de la Facultad de Cultura Física, Deporte y Recreación sede Bogotá. Magistro. U. Santo Tomás - Vicerrectoría de Universidad Abierta a Distancia. (Bogotá). 
11. MARCÉN MUÑ̃íO, C. 2007. El Deporte un Nuevo León. Un estudio sociológico desde una perspectiva de género. Disponible desde Internet en: www.efdeportes. com/efd117/EldeporteenNuevoLeon.pdf. (con acceso el 02/04/2010).

12. SARMIENTO, L.; VARGAS, H. 2002. El trabajo de las mujeres en Colombia. Corporación viva la ciudadanía. (Colombia). p.101-104.

13. SILVA CAMARGO, G. 2002. Diccionario básico del Deporte y la Educación Física. Kinesis. (Colombia). 267p.

14. SUAY I LERMA, F. 2003. El síndrome de sobreentrenamiento desde la psicología del deporte. Paidotribo. (España). 16p.
15. VRIJENS, J. 2006. Entrenamiento razonado del deportista. INDE. Barcelona (España). 499p.

16. WORCHEL, S.; COOPER, J.; GOETHALS, G.R.; OLSON, J.M. 2002. Prejuicios y estereotipos, Psicología social. Thompson Learning.

17. CONSEJO SUPERIOR DE DEPORTES. 2001. Las mujeres en la alta competencia deportiva. Estudios sobre ciencias del deporte. (España), 13 p.

Recibido: Enero 18 de 2012

Aceptado: Mayo 3 de 2012 